\title{
THE GENERALIZED DIVISOR PROBLEM AND THE RIEMANN HYPOTHESIS
}

\author{
HIDEKI NAKAYA
}

\section{Introduction}

Let $d_{z}(n)$ be a multiplicative function defined by

$$
\zeta^{z}(s)=\sum_{n=1}^{\infty} \frac{d_{z}(n)}{n^{s}} \quad(\sigma>1)
$$

where $s=\sigma+i t, z$ is a complex number, and $\zeta(s)$ is the Riemann zeta function. Here $\zeta^{z}(s)=\exp (z \log \zeta(s))$ and let $\log \zeta(s)$ take real values for real $s>1$. We note that if $z$ is a natural number $d_{z}(n)$ coincides with the divisor function appearing in the Dirichlet-Piltz divisor problem, and $d_{-1}(n)$ with the Möbious function.

The generalized divisor problem is concerned with finding an asymptotic formula for $\sum_{n \leq x} d_{z}(n)$, which was observed for real $z>0$ by A. Kienast [6] and K. Iseki [4] independently. A. Selberg [8] considered for all complex $z$, his result being

$$
D_{z}(x) \equiv \sum_{n \leq x} d_{z}(n)=\frac{x(\log x)^{z-1}}{\Gamma(z)}+O\left(x(\log x)^{\Re z-2}\right)
$$

uniformly for $|z| \leq A, x \geq 2$, where $A$ is any fixed positive number.

Next, let $\pi_{k}(x)$ be the number of integers $\leq x$ which are products of $k$ distinct primes. For $k=1, \pi_{k}(x)$ reduces to $\pi(x)$, the number of primes not exceeding $x$. C. F. Gauss stated empirically that $\pi_{2}(x) \sim x(\log \log x) / \log x$, and, by using the prime number theorem, E. Landau proved that $\pi_{k}(x) \sim$ $x(\log \log x)^{k-1} /(k-1) ! \log x$. Selberg considered $D_{z}(x)$ not only for its own sake but also with an intension to derive

$$
\pi_{k}(x)=\frac{x Q(\log \log x)}{\log x}+O\left(\frac{x(\log \log x)^{k}}{k !(\log x)^{2}}\right)
$$

uniformly for $1 \leq k \leq A \log \log x$, where $Q(x)$ is oplynomial of degree 
$k-1$.

In this paper we shall consider the connections between the asymptotic formulas of $D_{z}(x), \pi_{k}(x)$ and the location of zeros of the Riemann zeta function, thereby establishing some necessary and sufficient conditions for the truth of the Riemann hypothesis.

The main term of (1) and (2) is, however, inconvenient for our aim so that we introduce the following integrals as the main terms of $D_{z}(x)$ and $\pi_{k}(x)$ respectively:

$$
\begin{aligned}
\Phi_{z}(x) & =\frac{1}{2 \pi i} \int_{L} \zeta^{z}(s) \frac{x^{s}}{s} d s \\
F_{k, \varepsilon}(x) & =\frac{1}{(2 \pi i)^{2}} \int_{|z|=1} \int_{L \varepsilon} \zeta^{z}(s)\left\{\prod_{p}\left(1+\frac{z}{p^{s}}\right)\left(1-\frac{1}{p^{s}}\right)^{z}\right\} \frac{1}{z^{k+1}} \frac{x^{s}}{s} d s d z
\end{aligned}
$$

where $L_{\varepsilon}$ is, for every $\varepsilon$ and any $r(\varepsilon>0, r>0, \varepsilon+r<1 / 2)$, the path which begins at $1 / 2+\varepsilon$, moves to $1-r$ along the real axis, encircle the point 1 with radius $r$ in the counterclockwise direction, and returns to $1 / 2+\varepsilon$ along the real axis. The path $L$ is $L_{0}$.

The error terms are defined by

$$
\begin{aligned}
\Delta_{z}(x) & =D_{z}(x)-\Phi_{z}(x), \\
R_{k, \varepsilon}(x) & =\pi_{k}(x)-F_{k, \varepsilon}(x),
\end{aligned}
$$

Let $\Theta=\sup \{\sigma: \zeta(\sigma+i t)=0\}$.

Then the following Theorems 1 and 2 follow from more general results proved in Sections 1 and 2 below.

Theorem 1. We have

$$
\Delta_{z}(x) \ll x \exp \left(-c(\log x)^{3 / 5}(\log \log x)^{-1 / 5}\right)
$$

uniformly for $|z| \leq A, x \geq 3$.

Further we have

$$
\Delta_{z}(x) \ll x^{\theta+\varepsilon}
$$

uniformly for $|z| \leq A, x \geq 1$.

Conversely, if $\Delta_{z}(x) \ll x^{8+\varepsilon}$ for some $z \in C-Q^{+}$, where $Q^{+}$denotes the set of all non negative rational numbers, then, $\zeta(s)$ has no zeros for $\sigma>\Xi$.

Remark. 1. If we define $\alpha_{z}$ by

$$
\alpha_{z}=\inf \left\{\alpha: \Delta_{z}(x) \ll x^{\alpha}\right\}
$$

as in the Dirichlet-Piltz divisor problem, it is well known that the Lindelöf 
hypothesis is equivalent to $\alpha_{z} \leq 1 / 2$ for all natural number $z$. Theorem 1 however in this respect shows that $\alpha_{z} \leq 1 / 2$ for all complex $z$ under the Riemann hypothesis. Conversely, the Riemann hypothesis follows from $\alpha_{z} \leq 1 / 2$ for some $z \in C-Q^{+}$.

2. R. D. Dixon [3] gives an asymptotic expansion of $\Phi_{z}(x)$ in the form

$$
\Phi_{z}(x)=x(\log x)^{z-1} \sum_{m=0}^{N-1} \frac{B_{m}(z)}{(\log x)^{m} \Gamma(z-m)}+O\left(x(\log x)^{\Re z-N-1}\right)
$$

uniformly for $|z| \leq A$. Here $N$ is any fixed integer $\geq 1$ and $B_{m}(z)$ are regular functions of $z$, especially $B_{0}(z)=1$.

3. R. Balasubramanian and K. Ramachandra [1] have observed an asymptotic formula for $\sum_{n d(n) \leq x} 1$ by a method similar to that of Selberg [8] or H. Delange [2].

Theorem 2. We have

$$
R_{k, \varepsilon}(x) \ll x \exp \left(-c(\log x)^{3 / 5}(\log \log x)^{-1 / 5}\right)
$$

uniformly for $k \geq 1, x \geq 3$ with some constant $c$.

Further we have

$$
R_{k, \varepsilon}(x) \ll x^{\Theta+\varepsilon}
$$

uniformly for $k \geq 1, x \geq 1$.

Conversely, if $R_{k, \varepsilon}(x) \ll x^{\xi+\varepsilon}$ for some $k \geq 1$, $\zeta(s)$ has no zeros for $\sigma>\Xi$.

Remark. 1. If we define $r_{k}$ by

$$
r_{k}=\inf _{\varepsilon} \inf \left\{r: R_{k, \varepsilon}(x) \ll x^{r}\right\}
$$

Theorem 2 shows that $r_{k}=\Theta$ for every $k \geq 1$. That $\Theta=1 / 2$ is equivalent to the truth of the Riemann hypothesis.

2. H. Delange [2] gives an asymptotic expansion of $F_{k, \varepsilon}(x)$ in the form

$$
F_{k, \varepsilon}(x)=\frac{x}{\log x} \sum_{m=0}^{N-1} \frac{Q_{m}(\log \log x)}{(\log x)^{m}}+O\left(\frac{x(\log \log x)^{k-1}}{(\log x)^{N+1}}\right)
$$

for every $k \geq 1$, where $N$ is any fixed integer $\geq 1$ and $Q_{m}(x)$ are polynomials of degree not exceeding $k-1$.

3. Similar results hold for $\omega_{k}(x)$ and $\Omega_{k}(x)$. Here $\omega_{k}(x)\left(\Omega_{k}(x)\right)$ denotes the number of integers $\leq x$ which have $k$ distinct prime factors. (which have $k$ prime factors, allowing multiplicity.) 


\section{$\S 1$. The generalized divisor problem}

Actually we prove a more general statement than Theorem 1 and Theorem 2.

Suppose $f(s, z)=\sum_{n=1}^{\infty} b_{z}(n) n^{-s}$ is absolutely convergent for $\sigma>\frac{1}{2}$ and that $f(s, 0)=1$. We define the multiplicative function $a_{z}(n)$ is, as in Selberg [8], by $\zeta^{z}(s) f(s, z)=\sum_{n=1}^{\infty} a_{z}(n) n^{-s}$ for $\sigma>1$. Non negative number $\varepsilon_{0}$ is 0 or $\varepsilon$ according as $\lim _{s \rightarrow 1 / 2} f(s, z)<\infty$ or not, where $\varepsilon$ is arbitrary small positive number.

Lemma 1.1. We have

$$
\begin{aligned}
A_{z}(x) \equiv \sum_{n \leq x} a_{z}(n)= & \frac{1}{2 \pi i} \int_{L \varepsilon_{0}} \zeta^{z}(s) f(s, z) \frac{x^{s}}{s} d s \\
& +O\left(x \exp \left(-c(\log x)^{3 / 5}(\log \log x)^{-1 / 5}\right)\right)
\end{aligned}
$$

uniformly for $|z| \leq A, x \geq 3$.

Further, if we put $\Phi_{z, \varepsilon_{0}}(x)=(2 \pi i)^{-1} \int_{L \varepsilon_{0}} \zeta^{z}(s) f(s, z) s^{-1} x^{s} d x$, then $\Phi_{z, \varepsilon_{0}}(x)$ has the following asymptotic expansion

$$
\Phi_{z, \varepsilon_{0}}(x)=x(\log x)^{z-1} \sum_{m=0}^{N-1} \frac{B_{m}(z)}{(\log x)^{m} \Gamma(z-m)}+O\left(x(\log x)^{\Re z-N-1}\right)
$$

uniformly for $|z| \leq A$. Here $N$ is any fixed integer $\geq 1, B_{m}(z)$ are regular functions of $z$ to be defined in the proof, especially $B_{0}(z)=f(1, z)$.

Proof. The proof goes on the similar lines as those of $\mathrm{H}$. Delange [2], using the zero free region due to Vinogradov-Korobov.

We start from the expression

$$
\int_{0}^{x} A_{z}(u) d u=\lim _{T \rightarrow \infty} \frac{1}{2 \pi i} \int_{2-i T}^{2+i T} \zeta^{z}(s) f(s, z) \frac{x^{s+1}}{s(s+1)} d s .
$$

By Cauchy integral theorem, the path of integration can be deformed to $\sum_{i=1}^{7} L_{i}$ which are defined as follows:

$L_{1}$ is the segment $[2-i T, 1-\eta(-T)-i T]$,

$L_{2}$ is the curve $s=1-\eta(t)+i t\left(-T \leq t \leq-t_{0}\right)$ and two segments $\left[\eta-i t_{0}, \eta-i(1-\eta) \tan \delta\right]+\left[\eta-i(1-\eta) \tan \delta, 1 / 2+\varepsilon_{0}-i\left(1 / 2-\varepsilon_{0}\right) \tan \delta\right]$,

$L_{3}$ is the segment $\left[1 / 2+\varepsilon_{0}-i\left(1 / 2-\varepsilon_{0}\right) \tan \delta, 1+r e^{-i(\pi-\delta)}\right]$,

$L_{4}$ is the arc $s=1+r e^{i \theta}(-(\pi-\delta) \leq \theta \leq \pi-\delta)$,

$L_{5}$ is the segment $\left[1+r e^{i(\pi-\delta)}, 1 / 2+\varepsilon_{0}+i\left(1 / 2-\varepsilon_{0}\right) \tan \delta\right]$,

$L_{6}$ is two segments $\left[1 / 2+\varepsilon_{0}+i\left(1 / 2-\varepsilon_{0}\right) \tan \delta, \eta+i(1-\eta) \tan \delta\right]+$ 
$\left[\eta+i(1-\eta) \tan \delta, \eta+i t_{0}\right]$ and the curve $s=1-\eta(t)+i t\left(t_{0} \leq t \leq T\right)$,

$L_{7}$ is the segment $[1-\eta(T)+i T, 2+i T]$.

Here $\eta(t)=c(\log |t|)^{-2 / 3}(\log \log |t|)^{-1 / 3}, \eta=1-\eta\left(t_{0}\right)$ and $t_{0}$ is sufficiently large number to make $1 / 2<\eta<1$. Any positive numbers $\varepsilon, r$, and $\delta$ are satisfying $1 / 2+\varepsilon \leq \eta<1-r, 0<(1-\eta) \tan \delta<t_{0}$.

The contributions from the integral along $L_{1}+L_{2}+L_{6}+L_{7}$ are seen to give the error term, while the integral along $L_{3}+L_{4}+L_{5}$ gives the main term since that path becomes $L_{\varepsilon_{0}}$ allowing $\delta \downarrow 0$.

Regular functions $B_{m}(z)$ are defined as Taylor coefficients

$$
\{(s-1) \zeta(s)\}^{z} f(s, z) s^{-1}=\sum_{m=0}^{N-1} B_{m}(z)(s-1)^{m}+R_{N}(s, z) .
$$

LEMma 1.2. We have

$$
\log \zeta(s) \ll(\log |t|)^{1+2 \theta-2 \sigma+\varepsilon}
$$

uniformly for $\Theta<\sigma_{0} \leq \sigma \leq 1,|t| \geq 2$.

Proof. This is only a slight generalization of Theorem 14.2 in Titch$\operatorname{marsh}[9]$.

We define the error term $\Delta_{z, \varepsilon_{0}}(x)=A_{z}(x)-\Phi_{z, \varepsilon_{0}}(x)$, and let

$$
\alpha_{z}=\inf _{\varepsilon_{0}} \inf \left\{\alpha: \Delta_{z, \varepsilon_{0}}(x) \ll x^{\alpha}\right\}
$$

THEOREM 1.3. We have

$$
\alpha_{z} \leq \Theta
$$

for any $z \in C$.

Proof. We have $a_{z}(n) \ll n^{i+\varepsilon}$. Hence, by Lemma 3.12 in Titchmarsh [9], $A_{z}(x)$ has the expression,

$$
A_{z}(x)=\frac{1}{2 \pi i} \int_{2-i T}^{2+i T} \zeta^{z}(s) f(s, z) \frac{x^{s}}{s} d s+O\left(\frac{x^{2+\varepsilon}}{T}\right)
$$

uniformly for $T \geq 1$.

Let $\eta$ be a constant such that $\Theta<\eta<1$, and $\varepsilon, r$ and $\delta$ are any positive numbers such that $1 / 2+\varepsilon \leq \eta<1-r, 0<(1-\eta) \tan \delta<1$. Then the path of integration can be replaced by $\sum_{i=1}^{7} L_{i}$ which are defined as follows :

$L_{1}$ is the segment $[2-i T, \eta-i T]$, 
$L_{2}$ consists of two segments $[\eta-i T, \eta-i(1-\eta) \tan \delta]$

$$
+\left[\eta-i(1-\eta) \tan \delta, 1 / 2+\varepsilon_{0}-i\left(1 / 2-\varepsilon_{0}\right) \tan \delta\right],
$$

$L_{3}, L_{4}$ and $L_{5}$ are the same as in Lemma 1.1,

$L_{6}$ consists of two segments

$$
\begin{aligned}
{\left[1 / 2+\varepsilon_{0}+i\left(1 / 2-\varepsilon_{0}\right) \tan \delta\right.} & \eta+i(1-\eta) \tan \delta] \\
+ & {[\eta+i(1-\eta) \tan \delta, \eta+i T], }
\end{aligned}
$$

$L_{7}$ is the segment $[\eta+i T, 2+i T]$.

As in Lemma 1.1, $L_{3}+L_{4}+L_{5}$ becomes $L_{\varepsilon_{0}}$ by allowing as $\delta \downarrow 0$.

From Lemma 1.2, we have

$$
\zeta^{z}(s) f(s, z) \ll|t|^{\varepsilon}
$$

for $s \in L_{1}, L_{2}, L_{6}, L_{7}$. Therefore,

$$
\begin{gathered}
\int_{L_{1}}+\int_{L_{7}} \ll \int_{\eta}^{2} T^{\varepsilon} \frac{x^{2}}{T} d \sigma \ll T^{\varepsilon-1} x^{2}, \\
\int_{L_{2}}+\int_{L_{6}} \ll \int_{0}^{T} t^{\varepsilon} \frac{x^{\eta}}{t+1} d t+\int_{1 / 2+\varepsilon_{0}}^{\eta} x^{\eta} d \sigma \ll T^{\varepsilon} x^{\eta} .
\end{gathered}
$$

Hence

$$
A_{z}(x)=\Phi_{z, \varepsilon_{0}}(x)+O\left(T^{\varepsilon-1} x^{2}\right)+O\left(T^{\varepsilon} x^{\eta}\right)+O\left(\frac{x^{2+\varepsilon}}{T}\right) O\left(x^{\varepsilon}\right) .
$$

By taking $T=x^{2}, \eta=\Theta+\varepsilon$ we have

$$
A_{z}(x)=\Phi_{z, \varepsilon_{0}}(x)+O\left(x^{\theta+3 \varepsilon}\right) .
$$

This proves the theorem.

Theorem 1.4. We have

$$
\Theta \leq \alpha_{z}
$$

for any $z \in C-Q^{+}$.

Proof. First, we suppose that $\sigma>2$. Then,

$$
\begin{aligned}
s \int_{1}^{\infty} & \frac{\Phi_{z, \varepsilon_{0}}(x)}{x^{s+1}} d x=s \int_{1}^{\infty}\left(\frac{1}{2 \pi i} \int_{L \varepsilon_{0}} \zeta^{z}(\omega) f(\omega, z) \frac{x^{\omega}}{\omega} d \omega\right) \frac{1}{x^{s+1}} d x \\
& =\frac{s}{2 \pi i} \int_{L \varepsilon_{0}} \zeta^{z}(\omega) f(\omega, z) \omega^{-1}\left(\int_{1}^{\infty} x^{\omega-s-1} d x\right) d \omega \\
& =\frac{s}{2 \pi i} \int_{L \varepsilon_{0}} \zeta^{z}(\omega) f(\omega, z)\{\omega(s-\omega)\}^{-1} d \omega \\
& =\frac{1}{2 \pi i} \int_{L \varepsilon_{0}} \zeta^{z}(\omega) f(\omega, z) \omega^{-1} d \omega+\frac{1}{2 \pi i} \int_{L \varepsilon_{0}} \zeta^{z}(\omega) f(\omega, z)(s-\omega)^{-1} d \omega .
\end{aligned}
$$


The interchange of the order of integration is justified because of the absolute convergence. Hence

$$
\begin{aligned}
\zeta^{z}(s) f(s, z)= & s \int_{1}^{\infty} \frac{A_{z}(x)}{x^{s+1}} d x \\
= & s \int_{1}^{\infty} \frac{\Phi_{z, \varepsilon_{0}}(x)}{x^{s+1}} d x+s \int_{0}^{\infty} \frac{\Delta_{z, \varepsilon_{0}}(x)}{x^{s+1}} d x \\
= & \frac{1}{2 \pi i} \int_{L \varepsilon_{0}} \zeta^{z}(\omega) f(\omega, z) \omega^{-1} d \omega \\
& +\frac{1}{2 \pi i} \int_{L \varepsilon_{0}} \zeta^{z}(\omega) f(\omega, z)(s-\omega)^{-1} d \omega+s \int_{0}^{\infty} \frac{\Delta_{z, \varepsilon_{0}}(x)}{x^{s+1}} d x
\end{aligned}
$$

We put

$$
L_{\sigma_{0}}=\left\{s: \sigma_{0}<\sigma\right\}-\{s:|s-1| \leq r\}-\{s: t=0, \sigma<1\}
$$

for $1 / 2 \leq \sigma_{0} \leq 2$. Then on the right hand side of (3), the first and the second term can be continued analytically for $s \in L_{1 / 2}$ as a function of $s$, while the third term can be continued for $s \in L_{\alpha_{z}}$ since the involved integral converge uniformly for $\sigma>\alpha_{z}$. On the other hand the left hand side of (3) has singularities at the zeros of $\zeta(s)$ when $s \in C-Q^{+}$. We therefore conclude that $\Theta \leq \alpha_{z}$ for any $z \in C-Q^{+}$.

Remark. If we suppose that all the zeros of $\zeta(s)$ are simple, Theorem 1.4 holds for all $z \in C-N$.

Now Theorem 1 follows by taking $f(s, z) \equiv 1$.

\section{§2. The asymptotic formula for $\pi_{k}(x)$}

Throughout this section, we suppose that $a_{z}(n)$ is regular for $|z| \leq A$, and has a Taylor expansion at $z=0$ such that $a_{z}(n)=\sum_{k=0}^{\infty} c_{k}(n) z^{k}$ for $|z| \leq A$ with $A>1$.

LEMMA 2.1. We have

$$
\begin{aligned}
C_{k}(x) \equiv \sum_{n \leq x} c_{k}(n)= & \frac{1}{2 \pi i} \int_{|z|=1} \frac{\Phi_{z, \varepsilon_{0}}(x)}{z^{k+1}} d z \\
& +O\left(x \exp \left(-c(\log x)^{3 / 5}(\log \log x)^{-1 / 5}\right)\right)
\end{aligned}
$$

uniformly for $k \geq 1, x \geq 3$.

Further, if we put $F_{k, \varepsilon_{0}}(x)=(2 \pi i)^{-1} \int_{|z|=1} \Phi_{z, \varepsilon_{0}}(x) z^{-k-1} d z$, then $F_{k, \varepsilon_{0}}(x)$ has the following asymptotic expansion 


$$
F_{k, \varepsilon_{0}}(x)=\frac{x}{\log x} \sum_{m=0}^{N-1} \frac{Q_{m}(\log \log x)}{(\log x)^{m}}+O\left(\frac{x(\log \log x)^{k-1}}{(\log x)^{N+1}}\right)
$$

for every $k \geq 1$, where $Q_{m}(x)$ are polynomials of degree not exceeding $k-1$.

Proof. Since $A_{z}(x)$ is regular for $|z| \leq A$ as a function of $z$, and $C_{k}(x)$ is the coefficient of $z^{k}$, it follows by using Lemma 1.1 that

$$
\begin{aligned}
C_{k}(x) & =\frac{1}{2 \pi i} \int_{|z|=1} \frac{A_{z}(x)}{z^{k+1}} d z \\
& =\frac{1}{2 \pi i} \int_{|z|=1} \frac{\Phi_{z, \varepsilon_{0}}(x)}{z^{k+1}} d z+\frac{1}{2 \pi i} \int_{|z|=1} \frac{\Delta_{z, \varepsilon_{0}}(x)}{z^{k+1}} d z
\end{aligned}
$$

where

$$
\begin{aligned}
\frac{1}{2 \pi i} \int_{|z|=1} \frac{\Delta_{z, \varepsilon_{0}}(x)}{z^{k+1}} d z & \ll \max _{|z|=1}\left|\Delta_{z, \varepsilon_{0}}(x)\right| \\
& \ll x \exp \left(-c(\log x)^{3 / 5}(\log \log x)^{-1 / 5}\right)
\end{aligned}
$$

which proves the first half. The proof of the second half can be found in H. Delange [2].

Remark. For $k=1$, we can express the main term in terms of the logarithmic integral. Namely, starting from the expression

$$
\Phi_{z, \varepsilon_{0}}(x)=\int_{2}^{x}\left(\frac{1}{2 \pi i} \int_{L \varepsilon_{0}} \zeta^{z}(s) f(s, z) x^{s-1} d s\right) d x+O(1)
$$

and proceeding as in the proof of $H$. Delange [2] we obtain

$$
F_{1, \varepsilon_{0}}(x)=\int_{2}^{x} \frac{d u}{\log u}+O\left(x^{1 / 2+\varepsilon_{0}}\right)
$$

so that

$$
C_{1}(x)=\int_{2}^{x} \frac{d u}{\log u}+O\left(x \exp \left(-c(\log x)^{3 / 5}(\log \log x)^{-1 / 5}\right)\right) .
$$

This satisfies the assertion.

We define the error term $R_{k, \varepsilon_{0}}(x)=C_{k}(x)-F_{k, \varepsilon_{0}}(x)$, and let

$$
r_{k}=\inf _{\varepsilon_{0}} \inf \left\{r: R_{k, \varepsilon_{0}}(x) \ll x^{r}\right\} .
$$

Theorem 2.2. We have

$$
r_{k}=\Theta
$$

for any $k \geq 1$. 
Proof. From Theorem 1.3, we have

$$
R_{k}(x)=\frac{1}{2 \pi i} \int_{|z|=1} \frac{\Delta_{z, \varepsilon_{0}}(x)}{z^{k+1}} d z \ll \max _{|z|=1}\left|\Delta_{z, \varepsilon_{0}}(x)\right| \ll x^{\theta+3 \varepsilon} .
$$

Hence $r_{k} \leq \Theta$.

Conversely,

$$
\begin{aligned}
C_{k}(x) & =\frac{1}{2 \pi i} \int_{|z|=1} \frac{A_{z}(x)}{z^{k+1}} d z \\
& =\frac{1}{2 \pi i} \int_{|z|=1}\left(\frac{1}{2 \pi i} \int_{2-i \infty}^{2+i \infty} \zeta^{z}(s) f(s, z) \frac{x^{s}}{s} d s\right) \frac{1}{z^{k+1}} d z \\
& =\frac{1}{2 \pi i} \int_{2-i \infty}^{2+i \infty}\left(\frac{1}{2 \pi i} \int_{|z|=1} \frac{\zeta^{z}(s) f(s, z)}{z^{k+1}} d z\right) \frac{x^{s}}{s} d s \\
& =\frac{1}{2 \pi i} \int_{2-i \infty}^{2+i \infty} G_{k}(s) \frac{x^{s}}{s} d s, \text { say. }
\end{aligned}
$$

Here we have

$$
G_{k}(s)=\sum_{l=0}^{k} \frac{1}{l !(k-l) !}(\log \zeta(s))^{l} f^{(k-l)}(s, 0)
$$

where $f^{(n)}(s, z)$ means the $n$-th derivative of $f(s, z)$ with respect to $z$. It follows that $G_{k}(s)$ is regular for $s \in L_{\theta}$, and has the expression $G_{k}(s)=$ $\sum_{n=1}^{\infty} c_{k}(n) n^{-s}$ for $\sigma>1$.

Thus

$$
F_{k, \varepsilon_{0}}(x)=\frac{1}{2 \pi i} \int_{L \varepsilon_{0}} G_{k}(s) \frac{x^{s}}{s} d s .
$$

If we suppose $\sigma>2$, then

$$
\begin{aligned}
s \int_{1}^{\infty} \frac{F_{k, \varepsilon_{0}}(x)}{x^{s+1}} d x & =s \int_{1}^{\infty}\left(\frac{1}{2 \pi i} \int_{L \varepsilon_{0}} G_{k}(\omega) \frac{x^{\omega}}{\omega} d \omega\right) \frac{1}{x^{s+1}} d x \\
& =\frac{s}{2 \pi i} \int_{L \varepsilon_{0}} G_{k}(\omega) \omega^{-1}\left(\int_{1}^{\infty} x^{\omega-s-1} d x\right) d \omega \\
& =\frac{1}{2 \pi i} \int_{L \varepsilon_{0}} G_{k}(\omega) \omega^{-1} d \omega+\frac{1}{2 \pi i} \int_{L \varepsilon_{0}} G_{k}(\omega)(s-\omega)^{-1} d \omega .
\end{aligned}
$$

Hence

$$
\begin{aligned}
G_{k}(s)= & s \int_{1}^{\infty} \frac{C_{k}(x)}{x^{s+1}} d x=s \int_{1}^{\infty} \frac{F_{k, \varepsilon_{0}}(x)}{x^{s+1}} d x+s \int_{1}^{\infty} \frac{R_{k, \varepsilon_{0}}(x)}{x^{s+1}} d x \\
= & \frac{1}{2 \pi i} \int_{L \varepsilon_{0}} G_{k}(\omega) \omega^{-1} d \omega+\frac{1}{2 \pi i} \int_{L \varepsilon_{0}} G_{k}(\omega)(s-\omega)^{-1} d \omega \\
& +s \int_{1}^{\infty} \frac{R_{k, \varepsilon_{0}}(x)}{x^{s+1}} d x .
\end{aligned}
$$


Now on the right hand side of (4), the first and the second term can be continued analytically for $s \in L_{1 / 2}$ as a function of $s$, while the third term can be continued for $s \in L_{r_{k}}$, since the involved integral converges uniformly for $L_{r_{k}}$.

But $G_{k}(s)$ has singularities at zeros $\rho=\beta+i \gamma$, say, of $\zeta(s)$. In fact, if we consider the limit $G_{k}(\sigma+i \gamma)$ as $\sigma \downarrow \beta$, under the assumption that $\rho$ is a zero of order $M$,

$$
\begin{aligned}
G_{k}(\sigma+i \gamma) & \sim \sum_{l=0}^{k} \frac{1}{l !(k-l) !}(\log \zeta(\sigma+i \gamma))^{l} f^{(k-l)}(\sigma+i \gamma, 0) \\
& \sim \sum_{l=0}^{k} \frac{1}{l !(k-l) !}(M \log (\sigma-\beta))^{l} f^{(k-l)}(\rho, 0) \\
& \sim \sum_{l=0}^{k} \frac{1}{l !(k-l) !} M^{l}(-t)^{l} f^{(k-l)}(\rho, 0) \quad\left(\sigma-\beta=e^{-t}\right) \\
& \sim M^{k} t^{k}(t \rightarrow \infty),
\end{aligned}
$$

for $f^{(k-l)}(\rho, 0)$ is bounded and $f(s, 0)=1$. Hence we conclude that $\Theta \leq r_{k}$ for any $k \geq 1$.

Now Theorem 2 follows by taking

$$
\begin{array}{ll}
f(s, z)=\prod_{p}\left(1+\frac{z}{p^{s}}\right)\left(1-\frac{1}{p^{s}}\right)^{z} & \text { for } \pi_{k}(x) \\
f(s, z)=\prod_{p}\left(1+\frac{z}{p^{s}-1}\right)\left(1-\frac{1}{p^{s}}\right)^{z} & \text { for } \omega_{k}(x) \\
f(s, z)=\prod_{p}\left(1-\frac{z}{p^{s}}\right)^{-1}\left(1-\frac{1}{p^{s}}\right)^{z} & \text { for } \Omega_{k}(x)
\end{array}
$$

which satisfy the assumptions on $f(s, z)$ at the top of Sections 1 and 2 .

Acknowledgement. The author would like to thank Professors S. Egami and S. Kanemitsu for many valuable discussions and encouragements.

\section{REFERENCES}

[1] R. Balasubramanian and K. Ramachandra, On the number of integers $n$ such that $n d(n) \leq x$, Acta Arith., 49 (1988), 313-322.

[2] H. Delange, Sur des formules de Atle Selberg, Acta Arith., 19 (1971), 105-146.

[ 3 ] R. D. Dixon, On a generalized divisor problem, J. Indian Math., 28 (1964), 187-196.

[4] K. Iseki, On a divisor problem generated by $\zeta^{\alpha}(s)$, Natural Science Report, Ochanomizu Univ., 4.2 (1953), p. 175.

[5] A. Ivić, "The Riemann zeta function," Wiley Interscience, New-York, 1985.

[6] A. Kienast, Über die asymptotische darstellung der summatorischen funktion von 
Dirichletreihen mit positiven koeffizienten, Math. Z., 45 (1939), 554-558.

[7] E. Landau, "Handbuch der Lehre von der Verteilung der Primzahlen.," Chelsea, New-York, 1969.

[ 8 ] A. Selberg, Note on a paper by L. G. Sathe, J. Indian Math. 18 (1954), 83-87.

[9] E. C. Titchmarsh and D. R. Heath Brown, "The Theory of the Riemann ZetaFunction," Oxford University Press, Oxford, 1986.

Department of Mathematics

Kanazawa University

Kanazawa 920

Japan 